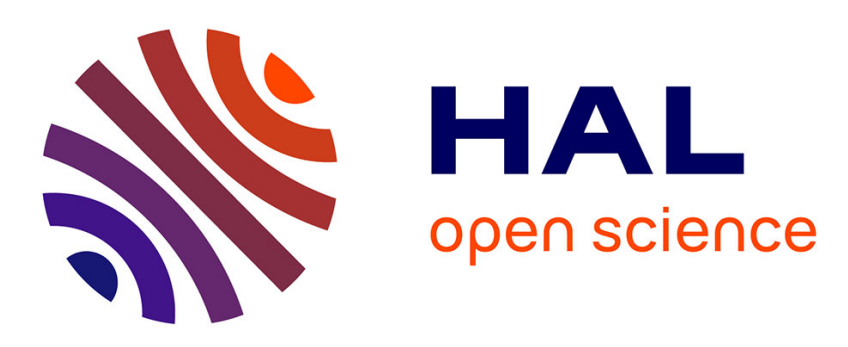

\title{
Spatially Addressed Supramolecular Nanowires: A Full Structural Characterization by GIWAXS
}

Melodie Galerne, Benoît Heinrich, Arnaud Hemmerle, Philippe Fontaine, Nicolas Giuseppone, Patrick Kékicheff

\section{- To cite this version:}

Melodie Galerne, Benoît Heinrich, Arnaud Hemmerle, Philippe Fontaine, Nicolas Giuseppone, et al.. Spatially Addressed Supramolecular Nanowires: A Full Structural Characterization by GIWAXS. ACS Applied Polymer Materials, 2021, 3 (2), pp.661-670. 10.1021/acsapm.0c00973 . hal-03373633

\section{HAL Id: hal-03373633 \\ https://hal.science/hal-03373633}

Submitted on 11 Oct 2021

HAL is a multi-disciplinary open access archive for the deposit and dissemination of scientific research documents, whether they are published or not. The documents may come from teaching and research institutions in France or abroad, or from public or private research centers.
L'archive ouverte pluridisciplinaire HAL, est destinée au dépôt et à la diffusion de documents scientifiques de niveau recherche, publiés ou non, émanant des établissements d'enseignement et de recherche français ou étrangers, des laboratoires publics ou privés. 


\title{
Spatially Addressed Supramolecular Nanowires:
}

\section{A Full Structural Characterization by GIWAXS}

\author{
Melodie Galerne, ${ }^{\mathrm{a}}$ Benoît Heinrich, ${ }^{\mathrm{b}}$ Arnaud Hemmerle, ${ }^{\mathrm{c}}$ Philippe Fontaine, ${ }^{\mathrm{c}}$ \\ Nicolas Giuseppone*a and Patrick Kékicheff*a,c
}

${ }^{a}$ Institut Charles Sadron, Université de Strasbourg, CNRS UPR22, 67034 Strasbourg, France

${ }^{b}$ Institut de Physique et Chimie des Matériaux de Strasbourg, Université de Strasbourg, CNRS UMR7504, 67034 Strasbourg, France

${ }^{c}$ Synchrotron SOLEIL, Saint Aubin, L’Orme des Merisiers, BP48, 91192 Gif-sur-Yvette, France

\author{
*Corresponding authors: \\ giuseppone@unistra.fr \\ patrick.kekicheff@ics-cnrs.unistra.fr
}

\begin{abstract}
Supramolecular nanowiring is based on the precise interconnection of nano- or microelectronic circuit elements with electroactive supramolecular polymers displaying directional charge transport properties. It is a particularly attractive topic of current research in order to access miniaturized organic electronic devices. The bottom-up construction of such integrated nanowires can be generated by specific supramolecular polymerization processes - based on addressed nucleation growth mechanisms - which provide a spatial precision in their placement that cannot be accessed by top-down approaches. However, to implement, quantify, and validate such
\end{abstract}


supramolecular interconnects in nanotechnologies, powerful characterization techniques, which probe both the fine structural and orientational features of the nanowires, are still required. Most techniques usually lack the ability to access the morphological parameters of the targeted nanostructures lodged within the electronic circuits at that scale (for instance between micro/nanoelectrodes). Even grazing-incidence wide-angle X-ray scattering (GIWAXS) poses major hurdles for the practical investigation of such miniaturized electronic devices due to beam path obstruction for most in-plane orientation of the samples. To overcome these limitations, we have implemented a sensing modality based on GIWAXS collection for unique sample orientation and spot shape analysis in reciprocal space allowing the entire film morphology to be accessed. In this case study involving triarylamine-based nanowires, we fully determine in a single experiment the packing structure of the supramolecular polymers interconnecting microelectrodes, together with their 3D and 2D orientational order parameters $\left(S_{3 \mathrm{D}} \approx 0.986\right.$ and $\left.S_{2 \mathrm{D}} \approx 0.91\right)$. Our investigation demonstrates that the nanowires lie not only flat along the substrate but are also aligned along the normal to the electrodes. Furthermore, this alignment occurs with a coherent length of more than $100 \mathrm{~nm}$, representing more than 200 molecular lengths along the nanowire axis. From a broader perspective, this work highlights the high potential of GIWAXS to become a reference method for achieving the full characterization of nanowiring processes with various types of supramolecular polymers and polymer self-assemblies integrated in complex device geometries.

KEYWORDS: supramolecular polymers; nanowires; supramolecular electronics; orientational alignment; microelectrodes; grazing incidence wide-angle X-ray scattering 


\section{INTRODUCTION}

One of the most challenging objectives in supramolecular electronics ${ }^{1-3}$ is to precisely interconnect circuit elements, such as nano- and micro-electrodes, with supramolecular organic nanowires. Because charge transport is highly directional, the entire device efficiency depends on both the (local) structural and (long range) orientational alignments of the organic (semi)conducting material. Hence, monodimensional supramolecular polymers with a high degree of internal organization (e.g. large number of aligned crystalline domains), and an optimal orientation between electrodes, are expected to considerably increase the charge transport efficiency and miniaturize organic electronic devices. ${ }^{3,4}$ This has been established for e.g. OLEDs, OFETS, ${ }^{5-8}$ solar cells, ${ }^{9-11}$ and chemical sensors. ${ }^{12,13}$

The long range anisotropy and orientational alignment of various kinds of supramolecular nanoobjects, such as fibers, rods, chains, and belts are most often studied by microscopy techniques, such as polarized optical microscopy, AFM, SEM, and TEM. ${ }^{14}$ In addition, spectroscopy techniques such as UV-vis absorption, spectroscopic ellipsometry (SE), and near-edge X-ray absorption fine structure (NEXAFS), are commonly used to probe the local organization of fibrillar structures. ${ }^{14,15}$ However, the precise molecular order of the supramolecular polymer backbone, from which the (nano)object is built-up, can only be probed and quantified by more local characterization techniques such as x-ray scattering. Though, when only a few supramolecular nanowires are arranged in an electronic circuit, the scattering experiment becomes more complex because of the low signal intensity and device geometry. We reasoned that, in principle, these limitations can be overcome by using grazing incidence wide-angle X-ray scattering (GIWAXS), as large areas within thin surficial layers are probed, with the advantage to elucidate the local molecular packing structure of the nanowires. ${ }^{16}$ Moreover, by measuring the sample in several directions, one can also potentially clarify the alignment and orientation of the polymer in their 
effective wiring configuration. ${ }^{17,18}$ Finally, the coherence length of the supramolecular polymers may be obtained, ${ }^{19}$ and a GIWAXS derived orientation parameter calculated. ${ }^{20}$ Therefore, one can expect that a single GIWAXS experiment could ideally provide a fully quantitative multiscale structural and orientational characterization of the supramolecular wires directly measured in the organic electronic device. Nevertheless, to the best of our knowledge, the necessary developments to implement such a powerful characterization method in the context of nanowired devices have not been investigated in the literature so far.

As a proof of principle, we decided to study the local structure and long-range anisotropy of self-assembled triarylamine-based supramolecular polymers ${ }^{21}$ placed between microelectrodes (Fig. 1). This new family of supramolecular polymers has been previously shown to display excellent electronic and photonic conducting properties. ${ }^{22-25}$ Their local supramolecular structures were characterized by various techniques, including powder X-ray diffraction (XRD). ${ }^{21}$ Moreover, we recently demonstrated the possibility to grow triarylamine (TAA) nanowires directly in between electrodes by introducing a so-called supramolecular electropolymerization process starting from TAA 1 (Fig. 1 ). ${ }^{26}$ The spatially addressed and oriented formation of these nanowires is accessible by a multistep oxidation / nucleation / growth mechanism (Fig. 1b-d). First, a solution of TAA 1 in heptane is drop casted onto interdigitated electrodes, while applying a direct current electric field of $1.25 \times 10^{7} \mathrm{~V} \cdot \mathrm{m}^{-1}$. Neutral TAA 1 molecules, that come in direct contact with the anode, are

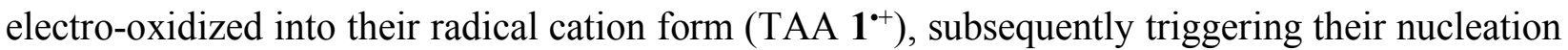
by charge transfer complexation and hydrogen bonding. The anode thus becomes a seeding surface from which the supramolecular polymers can grow: neutral TAA 1 stack onto the nuclei and the nanowires polymerize from the anode towards the cathode, following the lines of the applied electric field. Finally, the supramolecular polymers laterally aggregate with one another by van der Waals forces to produce larger bundles of supramolecular nanowires. This novel supramolecular 
electropolymerization process warrants unprecedented control over the localization of the interconnects, by $(i)$ addressing electro-oxidation of the TAA 1 monomers at the anode, and by $(i i)$ inducing directional growth of the supramolecular polymers in the applied electric field. The 2D orientational alignment of the supramolecular polymers was quantified for the direction normal to the electrodes, using SEM and AFM images, with order parameters of $0.87 \pm 0.09$ (at a concentration of $2 \mathrm{mg} / \mathrm{mL}$ ) and $0.94 \pm 0.08$ (at $5 \mathrm{mg} / \mathrm{mL}$ ). ${ }^{26}$ However, the 3D orientational parameter, as well as the local supramolecular structure and its coherence length are not accessible by these techniques but of first importance in the context of a nanowiring process.

We now present a much more powerful analysis by making use of a single GIWAXS experiment to fully characterize this system (Fig. 1e). The main challenge arises from the fact that a method, capable of delivering the local information within the electronic circuit, by directly characterizing the nanowires in between microelectrodes, is required in order to take optimal advantage of our systems configuration. Classical methods for deriving orientational order parameters, by means of rocking curves, cannot be used as the thin film has the same height $(\sim 100 \mathrm{~nm})$ as the microelectrodes, that consequently obstruct the path of the grazing incident beam. Furthermore, inplane rotation of the microelectronic device would inevitably lead to inherent variations, both in the effective scanned volumes and in the background scattering contributions made by the glass substrate. To overcome these experimental limitations, we resorted to a direct analysis of the intensities of Bragg reflections in the GIWAXS pattern. Here, we combine the radial and angular broadening observed for each spot in reciprocal space collected for the same configuration (incident beam parallel to the microelectrodes, $\omega=0$, Fig.1e) to infer the morphological parameters of the film. With this technique, not only the (supra)molecular organization of the nanowires can 
be determined, but, through the acquisition of their coherence length and both $2 \mathrm{D}$ and $3 \mathrm{D}$ orientational parameters, also their long-range orientation.

(a)

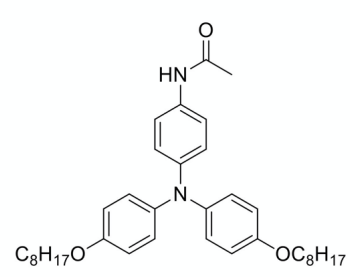

(c)

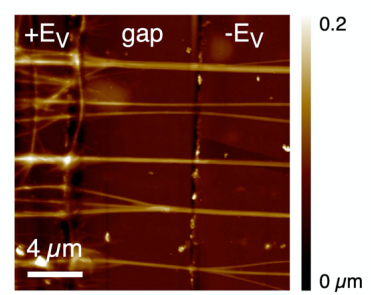

(d)

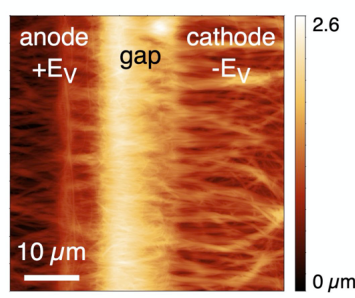

(b)

(e)
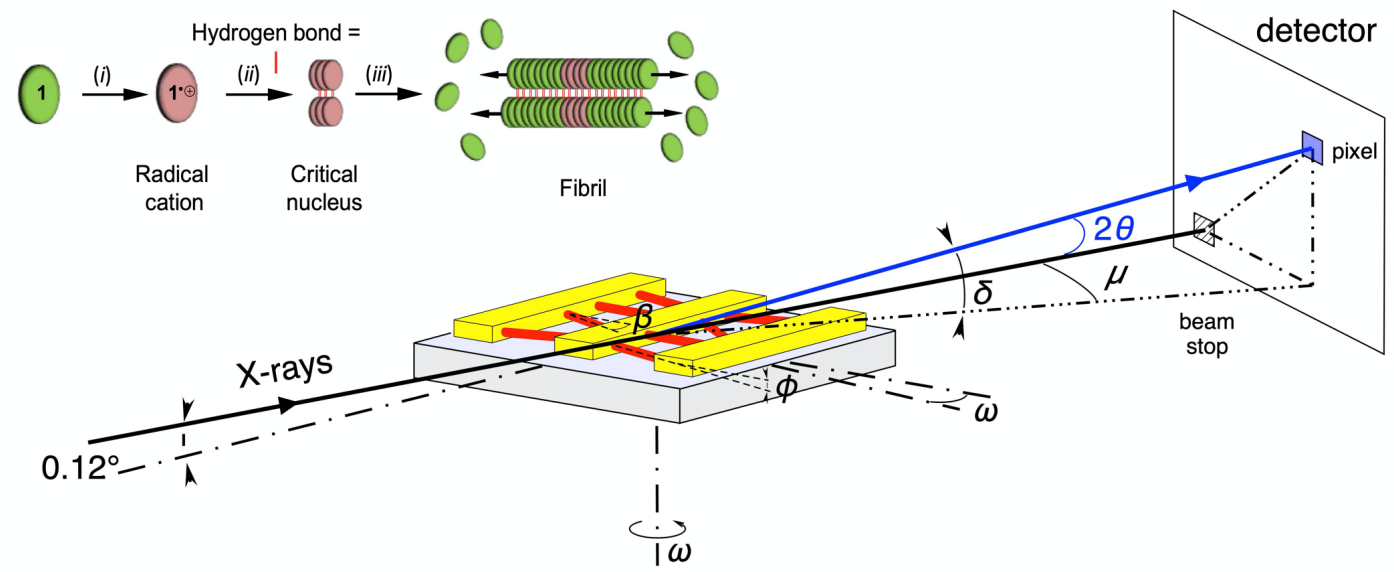

Figure 1. (a) Molecular structure of TAA 1; (b) Generic mechanism of supramolecular polymerization of TAA 1 through an oxidation / nucleation / growth process; (c and d) Atomic Force Microscopy (AFM) images of nanowires [reproduced from ref.26 with permission] formed by supramolecular electropolymerization of TAA 1 solutions in heptane at $2 \mathrm{mg} / \mathrm{mL}$ (c) and 5 $\mathrm{mg} / \mathrm{mL}$ (d); the solutions were drop casted onto $2.5 \mathrm{~mm}$ long interdigitated gold micro-electrodes (width $10 \mu \mathrm{m}$; height $100 \mathrm{~nm}$; spaced away by $10 \mu \mathrm{m}$ ) at a $1.25 \times 10^{7} \mathrm{~V} / \mathrm{m}$ applied DC electric field ${ }^{26}$; (e) Schematic of the GIWAXS setup on the SIRIUS beamline at the French National Synchrotron Radiation facility SOLEIL (Saint Aubin, France) ${ }^{27}$ including relevant geometry definitions. The sample is tilted by $0.12^{\circ}$ with respect to the incident beam for grazing incidence wide-angle measurements. The sample can be rotated about its vertical axis: its position is defined by the angle $\omega$ made by the normal to the microelectrodes with respect to the normal to the incident beam. In configuration where the incident beam is parallel to the microelectrodes $(\omega=0)$, all the electrode gaps, which are filled with TAA 1 nanowires, are irradiated simultaneously, as the x-ray footprint is $500 \mu \mathrm{m}$ wide. The angles $\phi$ and $\beta$ define the disorientation of the long axis of a nanowire with respect to the substrate plane and to the normal to the electrodes respectively. The horizontal ("longitude") angle, $\mu$, and the vertical ("latitude") angle, $\delta$, define the direction of the diffracted beam with respect to the incident beam. The diffracted beam hits the 2D detector (detector rotation not shown) with a total scattering angle $2 \theta$ with $\theta$ the Bragg angle. 


\section{RESULTS AND DISCUSSION}

Structure of the supramolecular nanowires films. The GIWAXS patterns, collected at low (2.5 $\mathrm{mg} / \mathrm{mL})$ and high concentration $(5 \mathrm{mg} / \mathrm{mL})$ of TAA 1 , once the electropolymerization was processed, are each composed of up to 40 reflections, going from 17 to $3 \AA$ in real space (see Fig. 2 and Fig.S1 in ESI $\dagger$ ). Through the examination of the positions of the diffraction spots and the averaging of the radial scattering, the structure of the supramolecular polymer film is resolved. Both concentrations lead to the same structural determination. The low-angle region is composed of (i) two intense first order reflections $\left(0.3525 \AA^{-1}\right.$ and $\left.0.3726 \AA^{-1}\right)$ and (ii) a series of weaker higher order reflections of $(h k 0)$ type, occurring from a bidimensional sub-lattice of rectangular symmetry. The long and short edges of this sub-lattice are assigned to the $a$ and $b$-axes, with $a=$

33.7(3) $\AA$ and $b=21.0(6) \AA$. The systematic missing $(h k 0)$ peaks with odd $h+k$ sum suggests a centered symmetry for the sub-lattice. ${ }^{28}$ The wide-angle region includes crossed reflections involving the periodicity along the $c$-axis, from which the three-dimensional structure is determined. The indexation converges on a crystal lattice of monoclinic symmetry. A simple centered 3D lattice cannot be brought into agreement with the measured sets of $q$ values, since some $(h k l)$ peaks with odd $h+k+l$ sum can be detected. Thus, the peak positions of all samples can be indexed to a monoclinic lattice according to:

$$
q_{\mathrm{hkl}}=2 \pi\left[\left(\frac{h}{a}\right)^{2}+\left(\frac{k}{b \sin \alpha}\right)^{2}+\left(\frac{l}{c \sin \alpha}\right)^{2}-2 \frac{k l}{b c} \frac{\cos \alpha}{\sin ^{2} \alpha}\right]^{1 / 2}
$$

with 3D lattice constants, $a=33.7(3) \AA, b=21.0(6) \AA$ and $c=9.7(0) \AA$, and angles $\alpha$ $=94 .(5)^{\circ}, \beta=\gamma=90^{\circ}$. 
(a)

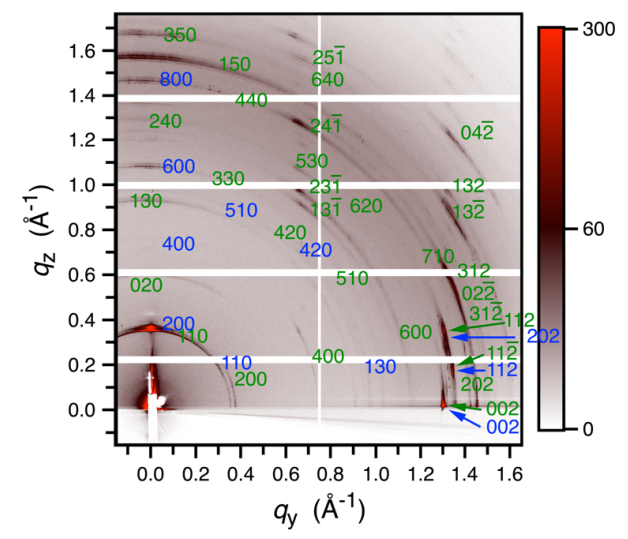

(b)
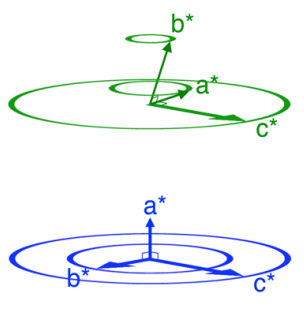

(c)

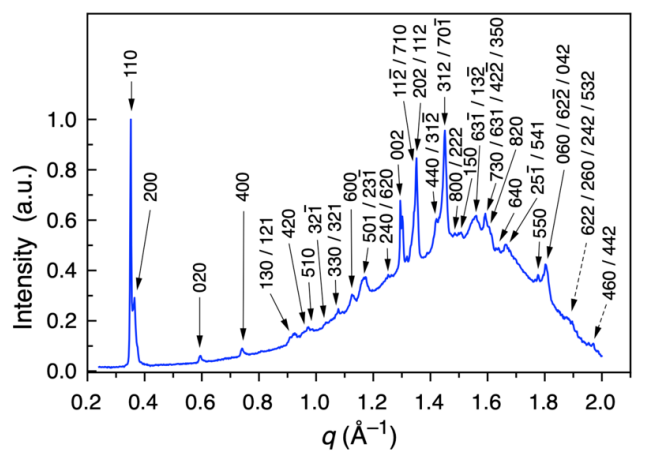

Figure 2. (a) GIWAXS pattern with the incident $x$-ray beam aligned parallel to the electrodes $(\omega=0$; Fig.1e), for an electropolymerized $2.5 \mathrm{mg} / \mathrm{mL}$ TAA 1 solution on interdigitated gold microelectrodes. The indexation of diffraction spots ( $h k l$ indices on the right of the spots) indicates two main preferential orientations (in green and in blue); (b) the corresponding orientations of reciprocal cell axes. The $c^{*}$-axis is oriented parallel to the substrate while the $a^{*}$-axis is either tilted by $0-30^{\circ}$ from (green) or normal to (blue) the substrate; (c) The indexed radial profile leads to a monoclinic cell with $a=33.7(3) \AA ; b=21.0(6) \AA ; c=9.7(0) \AA ; \alpha=94 .(5)^{\circ} ; \beta=\gamma=90^{\circ}$.

The volume of the unit cell is $6870 \AA^{3}$ and contains $Z=8$ molecules. Upon supramolecular electropolymerization, TAA $\mathbf{1}$ forms a crystalline structure of equivalent symmetry and a geometry that is close to the geometry of previously reported triarylamine monoamide molecules that selfassemble in solution after photooxidation. ${ }^{29}$ It is clearly evidenced here that supramolecular polymerization preserves the characteristic molecular shape and self-assembly by twinning of aryl segments through H-bonds into double-strands surrounded by a shell of aliphatic chains. Doublestrands then arrange at the nodes of rectangular columnar $\boldsymbol{a} \times \boldsymbol{b}$ sublattice with somewhat shrunken $a / b$ ratios, as compared to hexagonal geometry $(a / b=\sqrt{ } 3)$, and with a periodicity of two molecular thicknesses along the $c$-axis. Non-orthogonal angle $\alpha$ could here be determined, implying that the double-strands lie along the $b$-axis with a longitudinal shift of 1.6-1.7 $\AA$. 
A schematic representation of the molecular organization can be proposed from the GIWAXS analysis (Fig. 3). When piling, TAA 1 molecules present a colinear arrangement of their central nitrogen atoms separated by the distance $h_{\mathrm{mol}}$ of $0.5 \mathrm{~nm}$. Because of the propeller shape of the TAA units, their aromatic rings come at closer stacking contact which also enforces them to alternate their helical chirality between $\Delta$ and $\Lambda$ enantiomers. Because of the $60^{\circ}$ tilt of TAA along their elongation axis, the characteristic repeating unit $h$ is equal to $1 \mathrm{~nm}\left(2 \times h_{\mathrm{mol}}\right)$. Each monocolumn of TAA is laterally associated to another one by an infinite string of hydrogen bonds provided by the amide groups.

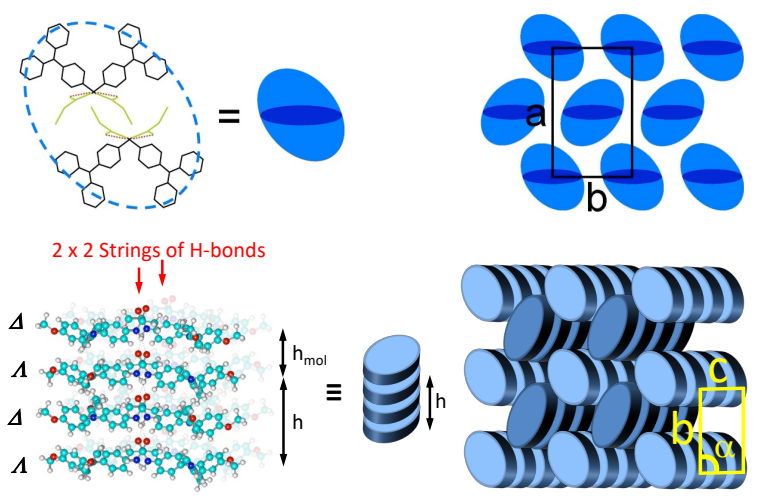

Figure 3. Schematic representations of the molecular organization. Left: association of triarylamine segments by $\mathrm{H}$ bonding and cross-section of double-strands; bottom left: structure of triarylamine double-strands from side and equivalent columns of piled molecular units, showing periodicity $h$ of two molecular thicknesses $h_{\text {mol }}$ along strand axis*. Right: Arrangement in the columnar plane according to the lattice geometry and systematic extinctions; bottom right: Longitudinal positioning of columns and monoclinic structure. The aliphatic chains fill the empty spaces between the columns.

Morphology of the supramolecular nanowires films. As part of a full-in-plane rotation scan available, GIWAXS patterns could be collected with the incident beam parallel and perpendicular to the direction of the electrodes, as illustrated in Fig. 1e. In the $90^{\circ}$ direction, the $\sim 100-150 \mathrm{~nm}$ high electrodes obstructs the path of the grazing incident beam, and only the superficial top film 
contributes to the scattering. However, this information is difficult to extract because it is hindered by a strong scattered background signal, due to the glass substrate in the range 1.2 to $1.6 \AA^{-1}$ (see ESI†). Indeed, at such incidence angle, the beam foot-print is about $50 \mathrm{~mm}$ long, whereas the effective supramolecular polymer film length is $450 \mu \mathrm{m}$ in this geometry (sample composed of 15 electrode pairs of $2.5 \mathrm{~mm}$ long, having each a width of $10 \mu \mathrm{m}$ and spaced away by $10 \mu \mathrm{m}$ ). Thereby, in the following, our focus is mainly on the configuration where the incident $\mathrm{x}$-ray beam is parallel to the microelectrodes $(\omega=0)$. In this geometry, the scattering patterns arise from the bulk of the supramolecular polymers grown in the 30 gaps between the 15 parallel interdigitated gold microelectrodes. With the incident beam parallel to the electrodes, the scattering vector $q$ probes diffraction perpendicular to the nanowires, both in the in-plane and out-of-plane directions. In the presented data, a coordinate system is defined for the scattering vector $q$ associated with the GIWAXS patterns, with $q_{\mathrm{z}}$ defined as the component of $q$ in the specular direction (perpendicular to the silica substrate) and $q_{\mathrm{y}}$ defined as the $q$ component parallel to the substrate.

Whatever the concentration, the (002) diffraction peak appears as a spot located on the $q_{\mathrm{y}}$ axis in the configuration where the incident x-ray beam is parallel to the microelectrodes (Fig.2a and Fig.S1). This indicates that the nanowires are strongly confined to an in-plane configuration. As it will be shown below, the sharpness of this peak suggests that the nanowires lie not only flat along this axis, but are also well aligned along the normal to the electrodes. These conclusions are further advocated by the absence of (002) spot when the samples are rotated by $90^{\circ}$ (Fig.S1), beyond the experimental limitations already mentioned. The $c$-axis is thus chosen to be oriented along the long-axis of the nanowires and the rectangular sublattice $\boldsymbol{a} \times \boldsymbol{b}$ is therefore in a plane almost orthogonal to the substrate (the angle $\alpha$ between the $b$-axis and the $c$-axis being $\left.\sim 94 .(5)^{\circ}\right)$. 
At low concentration, the arc-shaped reflections, observed in the GIWAXS pattern for all the out-of-plane directions (Fig.2a), indicate a rotational freedom of the crystal along its long $c$-axis in comparison with the lamellar stacking vector. However, at this concentration, the rings are not fully isotropic and the signal concentrates around the peak positions with limited angular distributions of the arcs. This suggests the domination of preponderant structural motifs with a "roll" of the crystals remaining moderate. Remarkably, at this concentration of nanowires, the GIWAXS pattern arises mainly from two preferential orientations of the crystals (Fig. 2): the rectangular sublattice $\boldsymbol{a} \times \boldsymbol{b}$, with a non-orthogonal angle $\alpha$ between the $b$-axis and $c$-axis $\left(\alpha=94.5^{\circ}\right)$, is oriented with either one direction tilted, or normal to the substrate. The relative intensities of the observed spots indicate that the distribution of the crystallites with the planes normal to the [110] direction of the monoclinic cell (spots in green in Fig.2a) is the preferred orientation, compared to the second orientation with reticular planes normal to the [100] direction (spots in blue). The two preferential orientations revealed at low concentration underline that the alignment allows the columns composing the crystals (Fig.3) to build the set of the more dense reticular planes along the glass substrate. These planes are those where the columns are the closest to each other. The reticular planes that are the most dense, are normal to the diagonal [110] of the rectangular sublattice $\boldsymbol{a} \times \boldsymbol{b}$. The planes parallel to the columns faces are less dense because they are packed in a staggered configuration. The fact that anisotropic objects tend to align their leading crystallographic planes along substrates, has been observed in many systems, including lyotropic liquid crystals which undergo topological transformations from cylinders to lamellae encompassing different structures, such as anisotropic ribbons and connected rods. ${ }^{30-32}$ Note that these preferential orientations are lost when the nanowires are grown in a more concentrated solution. Indeed, at higher concentrations almost uniquely isotropic rings are observed in the out-of-plane directions. The $a^{*}$ 
and $b^{*}$-axis now have random orientations, even though the $c^{*}$-axis remains parallel to the substrate and perpendicular to the electrode direction, Fig.S1, ESI $\dagger$ ). In contrast, at the lower concentration, arc-shaped reflections are discerned (Fig.2a). This observation can be interpreted twofold. At high concentration, the tighter molecular packing reduces the flexibility gradient along the growth direction of the nanowires and preferential orientations of the rectangular sublattice $\boldsymbol{a} \times \boldsymbol{b}$ are not favored at the interface with the substrate. Indeed, steric restrictions may manifest themselves as freedom for the nuclei to place with no special orientation. Thereby, the crystals will subsequently grow along their long $c$-axis with no preferential orientations. Additional considerations can be suggested. In particular, the loss of out-of-plane spots, with local reinforced intensity in their limited angular arc-shaped reflections, may be apparent only. Therefore, it is possible that some preferential orientations are still present in the vicinity of the substrate interface at high concentration, but the signal coming from these orientations is dominated by the bulk of the more concentrated supramolecular film.

Parallelism of the nanowires to the substrate. In the configuration where the incident beam is parallel to the microelectrodes ( $\omega=0$, Fig.1e), the GIWAXS patterns share the same (002) diffraction peak, located along the in-plane $q_{\mathrm{y}}$-axis (Fig.2a and Fig.S1, ESI $†$ ) for all concentrations. This spot is similar in position to the one found in the $(00 l)$ progression with even Miller indices, characteristic of the stacking of the aromatic rings composing the triarylamine molecules in a centro-symmetric unit cell. ${ }^{29} \mathrm{Here}$, for the TAA 1 disk-like core motif, the $d$-spacing is $9.7 \AA$. The localization of the (002) spot on the $q_{\mathrm{y}}$-axis indicates that the nanowires are strongly confined to an in-plane configuration. Its sharpness suggests that the crystals lie flat along this axis with little out-of-plane tilt. The angular width of this strong equatorial reflection allows us to determine the 
degree of orientation of crystallites along the $c$-axis. In order to estimate the fraction of nanowires that is aligned and the degree of alignment, the GIWAXS intensity is integrated along the $2 \theta$ axis and plotted as intensity $I$ versus azimuth $\phi$.

Figure 4 shows the profile for the (002) spot measured at a concentration of $2.5 \mathrm{mg} / \mathrm{mL}$ (see Fig.2). Gaussian and Lorentzian shape function can be differentiated by comparing the fits of the tail of the peak. A single Gaussian function does not reproduce the weak broad tail at the bottom of the Bragg singularity. Good fits of the profile are obtained using either the sum of two Gaussian functions or a Lorentzian function. As the value of the maximum intensity is uncertain at the precise equator location in Fig.2, a small uncertainty arises in the width of the profile. Nevertheless, all the fitting functions lead to a similar full width at half maximum of the $I_{002}(\Phi)$ profile: the FWHM is $3.3 \pm 0.2^{\circ}$, which means that the aligned crystals have a very small variation of their tilt angle with respect to the substrate.

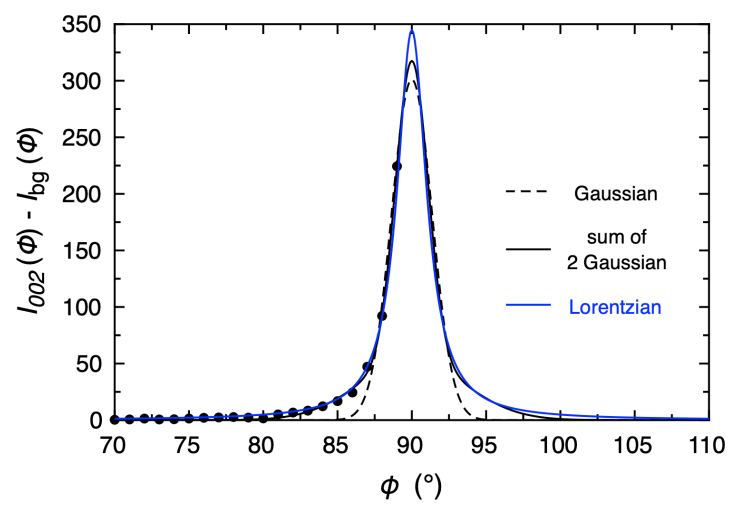

Figure 4. Azimuthal profile of the diffraction spot (002) after subtraction of the scattered background intensity, for the configuration in which the incident x-ray beam is oriented parallel to the micro-electrodes, and a concentration of $2.5 \mathrm{mg} / \mathrm{mL}$ is used (see Fig.2). The profile (solid circles) can be fitted using either a Lorentzian or the sum of two Gaussian functions. They all lead to a similar full width at half maximum (FWHM) of $3.3 \pm 0.2^{\circ}$. The uncertainty is estimated from the unknown maximum value that the intensity would take at the equator (the angle $\phi$ at exactly $90^{\circ}$ is out-of-reach in the GIWAXS pattern). 
The small spread of these lattice planes in the narrow out-of-plane distribution of the stacking peak about the surface normal (mosaic angle $\sim 1.6^{\circ}$ ) can be further quantified by calculating an orientational order parameter. For orientation distributions that are centrosymmetric as usually encountered for liquid crystals and polymer fibrils, the first non-vanishing moment of the angle $\phi$ between an element and the director is quadratic. The most common method to define the orientation of assemblies from x-ray scattering experiments is by calculating Herman's orientation parameter: ${ }^{33}$

$$
S_{3 \mathrm{D}}=\frac{1}{2}\left(3\left\langle\cos ^{2} \phi\right\rangle-1\right)
$$

where the mean-square cosine is calculated from the scattered intensity $I(\varphi)$ by integrating over the azimuthal angle $\varphi$ :

$$
\left\langle\cos ^{2} \phi\right\rangle=\frac{\int_{0}^{90} I(\varphi) \cos ^{2} \varphi \sin \varphi d \varphi}{\int_{0}^{90} I(\varphi) \sin \varphi d \varphi}
$$

For perfect horizontal orientation $S_{3 \mathrm{D}}=1$, while for isotropic orientation $S_{3 \mathrm{D}}=0$. Herman's orientation parameter refers to the second-order spherical harmonics of Legendre polynomial $P_{2}(\phi)$ of the cosine of that angle. Higher moments of the orientation distribution can also be obtained from the azimuthal profile of the reflection, and hence the full orientation distribution can be calculated, since the function can be expanded in series of even-order Legendre polynomials, ${ }^{34}$ with

$$
\left\langle P_{2 n}(\phi)\right\rangle=\frac{\int_{0}^{90} I(\varphi) P_{2 n}(\varphi) \sin \varphi d \varphi}{\int_{0}^{90} I(\varphi) \sin \varphi d \varphi}
$$

By applying equations 2-4 onto the azimuthal profile of the (002) spot shown in Fig.4, one obtains:

$$
S_{3 \mathrm{D}}=\left\langle P_{2}(\phi)\right\rangle=\frac{1}{2}\left\langle 3 \cos ^{2} \phi-1\right\rangle=0.986 \pm 0.002
$$

and 


$$
\left\langle P_{4}(\phi)\right\rangle=\frac{1}{8}\left\langle 35 \cos ^{4} \phi-30 \cos ^{2} \phi+3\right\rangle=0.951 \pm 0.006 .
$$

which is the second lowest and most important order parameter. These values demonstrate that the dominant feature of the GIWAXS patterns is a strong confinement of the supramolecular nanowires to a $2 \mathrm{D}$ plane with their long axis (i.e. the crystal $c$-axis) being parallel to the substrate: the nanowires lie flat on the substrate with negligible out-of-plane tilt. Taking advantage of this remarkable result, we will consider hereafter that the nanowires are positioned in the lateral plane of the substrate only.

Alignment of the nanowires along the normal to the microelectrodes. In this section the twodimensional disorientation of the nanowires is investigated. As already mentioned, the orientational distribution cannot be obtained by single rocking curves along a vertical axis, since accurate measurements of the diffracted signal is hampered by both variations in the footprint area and in the intensity of the grazing incident $\mathrm{x}$-ray beam due to path obstruction by the microelectrodes that have the same height as the film ( 100 nm height). To overcome these limitations, we resorted to the direct analysis of the intensities of Bragg reflections in the GIWAXS pattern. This approach makes use of the important constraint that the shapes of reflections at different positions on the detector are related for a given configuration (here, incident beam parallel to the microelectrodes). ${ }^{35}$ Indeed, the main sources of the finite size of the Bragg reflections are disorientation and finite crystallite sizes (in our synchrotron experiments the beam spread is negligible). The effects of finite crystallite size are directly related to the size of the reflection in reciprocal space while the effect of disorientation is to arc each reflection about the center of the diffraction pattern, forming part of a Debye-Scherrer ring. 
As the growth of the nanowires follows the lines of the applied electric field, we assume that the disorientation of their long axis is axisymmetric. Each nanowire is made of molecular segments, packed together to form well-ordered crystallites within each object. While the crystallites may have partial, weak, or no correlations in position and orientation, they nevertheless share the same common long axis of the nanowire in which they are embedded. This axis was defined conveniently as the $z$-axis in the real space. The $c$-lattice vector is the lattice vector parallel to that axis. In the following, the crystallites are considered to have a finite size and their average dimensions normal and parallel to the $z$-axis are noted $l_{\text {lat }}$ (i.e. in the lateral direction) and $l_{\text {axial }}$ (i.e. in the axial direction). The effects of finite crystallite size are directly related to the size of the reflection in reciprocal space (see also last section). The effect of disorientation is to rotate the reciprocal lattice point around the origin of reciprocal space, and the shape and width of the angular profile due to disorientation are approximately constant over most of reciprocal space.

If the substrate does not induce any preferential orientation, the crystallites are randomly positioned and randomly rotated about the $c$-axis. In such a case, the recorded diffraction pattern is that diffracted by a single crystallite object, cylindrically averaged about the $Z$ axis in reciprocal space. As a result, the positions of the Bragg reflections are described by the cylindrically projected reciprocal lattice onto the plane of the 2D-detector, provided the Ewald condition of diffraction is fulfilled. The cylindrical polar coordinates of the Bragg reflection in the reciprocal space are noted $\left(q_{i}, \sigma_{i}\right)$ with

$$
\cos \sigma_{i}=\frac{\overrightarrow{q_{l}} \cdot \overrightarrow{c^{*}}}{q_{i} c^{*}}
$$

The preferential orientations of the sublattice $\mathbf{a} \mathbf{x} \mathbf{b}$ induced by the substrate makes the projection to be not cylindrically isotropic. Combining this with the small variation in planar inclinations of the nanowire long axis to the direction normal to the microelectrodes, the superimposition of these 
two effects has the effect of spreading out the diffracted intensity from reflection along DebyeScherrer arcs. That is, the intensity at a given position of the detector is arced about the center of the pattern at constant diffraction angle. Following eqn.10 of the Methods section, and after corrections of the intensity and background subtraction, the profile of the $i^{\text {th }}$ reflection measured on the detector in polar coordinates $\left(q_{i}, \Phi_{i}\right)$ can be written in the form

$$
G_{i}(q, \phi)=I_{i} G_{q}\left(\frac{q-q_{i}}{\Delta q_{i}}\right) G_{\phi}\left(\frac{\phi-\phi_{i}}{\Delta \phi_{i}}\right)
$$

where $I_{i}$ is the peak intensity, $\Delta q_{i}$ and $\Delta \Phi_{i}$ are the radial and angular half-widths of the reflection of normalized profile shape $G_{q}$ and $G_{\Phi}$. The variations of the half-widths, with position on the detector, have been calculated. ${ }^{35}$ On the detector, placed at a distance $D$ from the sample rotated by an angle $\omega$ to the normal of the incident beam, these half-widths read:

$$
\Delta q_{i}=K \lambda D \frac{u\left|\sin \sigma_{i}\right|+v\left|\cos \sigma_{i}\right|}{\left(\left|\sin \sigma_{i}\right|+\left|\cos \sigma_{i}\right|\right)\left|\cos \theta_{i}\right| \cos ^{2} 2 \theta_{i}}
$$

and

$$
\Delta \phi_{i}=\beta+2 \pi \frac{u\left|\cos \sigma_{i}\right|+v\left|\sin \sigma_{i}\right|}{q_{i}\left(\left|\sin \sigma_{i}\right|+\left|\cos \sigma_{i}\right|\right)} \frac{\left|\sin \sigma_{i}\right|}{\sqrt{\left(\cos \theta_{i} \cos \omega\right)^{2}-\left(\cos \sigma_{i}-\sin \theta_{i} \sin \omega\right)^{2}}}
$$

where $\theta_{i}$ is the Bragg angle corresponding to the wavevector $q_{i}=(4 \pi / \lambda) \sin \theta_{i}$ in the reciprocal space at the wavelength $\lambda=1.24 \AA$ (energy $10 \mathrm{keV}$ ), and $K$ a calibration factor transforming pixel sizes of the detector into $\AA^{-1}$ units. In these expressions, the constants $u$ and $v$ are related to the mean crystallite sizes normal and parallel to the nanowire axis $\left(2 u=1 / l_{\text {lat }} ; 2 v=1 / l_{\text {axial }}\right)$, and $\beta$ to the mean disorientation.

As noted by Millane, ${ }^{35}$ the precise meaning of "mean" depends, of course, on the profile shape. In the following, the profiles of the Bragg reflections were fitted with Gaussian, Lorentzian, or pseudo-Voigt weighting functions. These shape functions can be differentiated by comparing the fits of the tails of the peaks. Nevertheless, they all lead to similar half-widths. The definitions of 
$\Delta q_{i}$ and $\Delta \Phi_{i}$ ensure that the same proportion of each reflection is included within the integration boundaries. Thereby, the inferred values from the fits are given with an uncertainty, including the small variation found when different models are used to describe the experimental profile shapes. It has also been noted ${ }^{35}$ that eqns. 7 and 8 are valid in the limit of small half-widths, which is the case here, as the analysis shows. On the other hand, the breadths of the reflections were found to be substantially larger than the beam size, as required by the method. Lastly, eqn. 8 is valid for small $\beta$ values, ${ }^{35,36}$ which is also found from the fits.

Thus, by examining the radial and angular profiles of the reflections in the configuration where the incident beam is parallel to the microelectrodes ( $\omega=0$ in eqn. 8 ), eqns. 7 and 8 have been used to estimate the three morphological parameters of the thin film, namely $l_{\text {lat }}, l_{\text {axial, }}$, and $\beta$. The focus of the analysis was on some well-resolved reflections to avoid interferences by overlapping profiles of adjacent reflections. In addition, the reflections located at large wavevectors allowed the most reliable results to be obtained, as the diffracted path is less obscured by the microelectrodes. For illustration, the collected intensity around the spots $(04 \overline{2})$ and $(13 \overline{2})$ arises from more than $90 \%$ of the sample volume contained in the gaps between the electrodes (total scattering angle $2 \theta>17^{\circ}$ ). For reflections with lower $q$ values, the diffraction spots located along the meridian of the detector were used as the blind zone is minimized. Note also that our analysis was carried out with most of the spots located away from the meridian axis and at relatively high $q$. Indeed, for an incident angle equal to the critical angle $\left(\alpha_{\mathrm{i}}=\alpha_{\mathrm{c}}\right)$ the patterns present a maximum in intensity localized at the exit angle $\alpha_{\mathrm{e}}=\alpha_{\mathrm{c}}$ (Yoneda's peak). ${ }^{37}$ This effect, well encountered upon reflectometry experiments, enhances the measured intensity close to meridian and to equator, principally at low $q$, but does not affect the other Bragg spots. So the potential residual contribution of the Yoneda's effect does not change our conclusions. 
A selection of angular and radial profiles is given in Figure 5. Good fits as well as less satisfactory fits for some arc reflections are deliberately illustrated to give an idea of the uncertainty obtained in the results. Nevertheless, the power of our procedure, when a whole set of arc reflections is investigated for the same GIWAXS configuration, is that the analysis converges to inferred morphological parameters of the film with low uncertainty. To our knowledge this direct method outperforms all other means for investigating thin films in complex geometries, such as films composed of nanowires placed between microelectrodes of microelectronic circuits. Thus, the mean crystallite sizes and disorientation were determined to be $l_{\text {lat }}=140 \pm 20 \AA, l_{\text {axial }}=1140 \pm$ $30 \AA$ and $\beta=12.2 \pm 0.5^{\circ}$. The low value found for the mean disorientation shows that the nanowires are aligned almost all parallel along the normal to the electrodes. The planar mosaïcity is $\pm 6^{\circ}$ and the distribution of the nanowires confined to the 2D plane have an orientational order parameter of

$$
S_{2 \mathrm{D}}=\left\langle 2 \cos ^{2} \beta-1\right\rangle=0.91 \pm 0.02
$$

This value is in good agreement with the order parameter inferred previously from SEM images. ${ }^{26}$ With $S_{3 \mathrm{D}} \approx 0.986$ (see above) and $S_{2 \mathrm{D}} \approx 0.91$ the nanowires do not only lie flat onto the substrate, but are also aligned parallel to the normal to the electrodes. This confirms the growth mechanism by supramolecular electropolymerization along the current lines of the electric field as reported previously. ${ }^{26}$

(a)

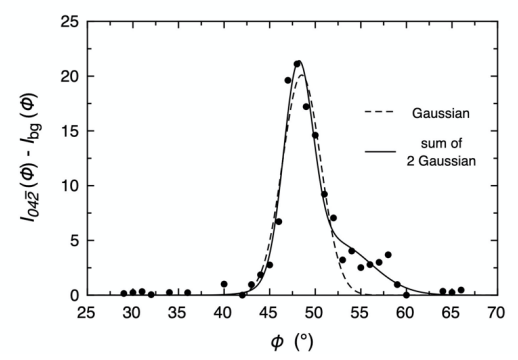

(b)

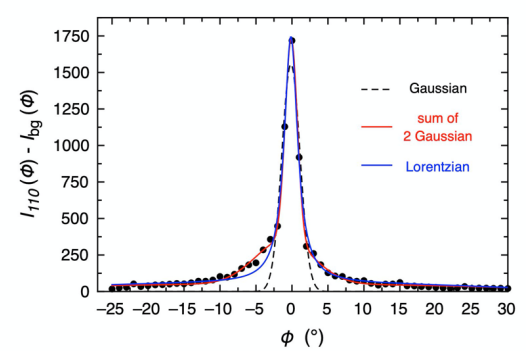

(c)

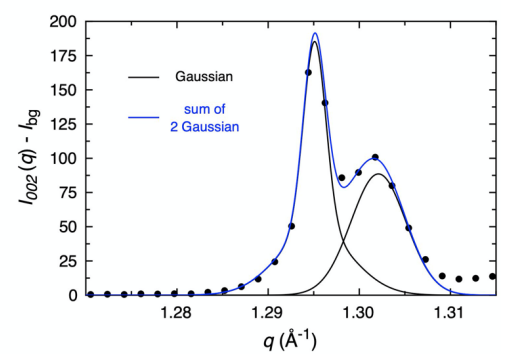

Figure 5. Angular (a,b) and radial (c) profiles of some diffraction spots after subtraction of the scattered background intensity, for the configuration in which the incident $\mathrm{x}$-ray beam is oriented 
parallel to the micro-electrodes, and a concentration of $2.5 \mathrm{mg} / \mathrm{mL}$ is used (see Fig.2). The angular profiles (solid circles) can be fitted using (a) the sum of two Gaussian functions for the spot $(04 \overline{2})$ (full width at half maximum (FWHM) is $5.0 \pm 0.3^{\circ}$ ) or (b) the sum of two Gaussian functions or a Lorentzian for the spot (110) with FWHM $=3.1 \pm 0.2^{\circ}$. (c) The radial profile (solid circles) of the diffraction spot (002) as a function of the wavevector $q$ is composed of two peaks centered respectively at $q=1.2951 \AA^{-1}$ and $1.3021 \AA^{-1}$. The presence of these overlapping peaks arises from the two preferential orientations of the nanowires on the substrate (in green and in blue; see Fig.2), but also to the imperfections of the sample: depending on the position of the diffracting zone, either upstream or downstream, the apparent $q$ values are slightly different. The profile can be fitted using the sum of two Gaussian functions of full width at half maximum (FWHM) $=0.0035 \AA^{-1}$ and $0.0071 \AA^{-1}$ respectively.

Crystallite sizes and coherence length. Analysis of very long anisotropic objects, such as fiber diffraction patterns, has pointed out that the "coherent molecular length" is a better concept than the axial crystallite size. ${ }^{38}$ Thus, the average length of the ordered crystallites along the long-axis of the nanowires is referred to as the coherence length. The intensity collected by spots, located along the equator of the detector, results from the layer planes of periodicity $c$ in the real space and distant by $l /(c \sin \alpha)$ in the reciprocal space. Equation 7 can be rewritten by introducing the average coherence length of the molecules along the nanowire long axis, $L_{c}$, and using the Scherrer formula relating the breadth, or full width at half-maximum, of the diffraction spot (002), with respect to the wavevector to the average axial size of the crystallite along the nanowires:

$$
L_{c}=2 \pi K_{\mathrm{Sch}} / \Delta q_{002}
$$

where the Scherrer constant, $K_{\mathrm{Sch}}$, has a value of about $0.9 .{ }^{38,39}$ The intensity profile of the spot (002) along the equator of the detector (i.e. $q_{\mathrm{x}}$-axis of Fig.2) is shown in Fig.5c. By applying eqn.9 one finds $L_{c} \approx 1200 \AA$. This value is in good agreement with the axial crystallite size $l_{\text {axial }}=1140$ $\pm 30 \AA$ inferred from the fits of the breadths of the other reflections (eqns.7 and 8). This remarkable length represents more than 200 molecular lengths along the long axis of the nanowires, grown by electropolymerization. Concerning the lateral crystallite size, $l_{\text {axial }}=140 \pm 20 \AA$, the inferred value 
from the fits appears larger than some narrow nanowires, that can be observed by SEM and AFM. We note however that these techniques are more sensitive to a number-average rather than a weight-average as in $\mathrm{x}$-ray techniques.

\section{New information provided by GIWAXS on nanowire devices prepared by} electropolymerisation. To sum up, the new type of GIWAXS analysis presented above is suitable to fully characterize TAA-based supramolecular nanowires directly electropolymerized between microelectrodes. The new information which is obtained compared to the more classical methods of analysis in the literature ${ }^{26}$ is manifold and includes: i) the unequivocal determination of the full set of crystallographic cell parameters and the modelling of the molecular organization; ii) the specification of the orientation of the columnar structure with respect to surface and with respect to electrodes; iii) the quantification of the distribution of domain orientations with two order parameters; iv) the estimation of the correlation lengths along nanowires and in the cross-sectional plane. This rich information is obtained while the nanowires could only be probed in a single sample orientation due to the presence of electrodes obstructing the beam. All the parameters accessed here cannot be determined to date by other techniques, and they are all of crucial importance to further probe structure-function relationships in organic electronic devices.

\section{CONCLUSION}

In conclusion, we have demonstrated the power of the GIWAXS technique to fully characterize the structural and orientational parameters of supramolecular nanowires grown in situ in organic electronic devices. This was particularly challenging as the nanowires remain inserted into the microelectronic circuits. By taking TAA-based supramolecular polymers as an example, we have been able to confirm their molecular structure when trapped between interdigitated electrodes. In 
addition, we have determined their coherence length in such a configuration $(>100 \mathrm{~nm})$. This is a quantitative result which remains inaccessible so far by other techniques. For instance, defects were sought by microscopy techniques such AFM. This was only possible on very large and hard monocrystals of triarylamines, and obviously only at their surfaces. Here, it was impossible to get this information by probing a very thin and soft nanowires configuration. Even if the fibers look perfect on large distances, information is lost on their internal structure. Therefore, it is a strong asset of the GIWAXS technique to access information that is not available in real space. Finally, both 3D and 2D orientation parameters have been determined, which is, once again, a clear advantage compared to microscopic analyses that are useful only for $2 \mathrm{D}$ parameters. With a remarkable precision, we demonstrate that the nanowires align not only parallel to the substrate, but equally along the current lines of the electric fields between the microelectrodes. As a whole,

our results demonstrate that a single GIWAXS experiment can provide all the necessary quantitative information to characterize in situ the most important structural and orientational features that are needed to characterize supramolecular electronic devices. If this study also reinforces our previous claims on supramolecular electropolymerization, ${ }^{26}$ it highlights more importantly the high potential of GIWAXS to become a reference method for achieving the full characterization of nanowiring processes involving various types of supramolecular polymers and polymer self-assemblies in complex geometries. With information that cannot be obtained by other means, our method could foster new supramolecular wiring approaches to be implemented, quantified, and validated in nanotechnologies.

\section{EXPERIMENTAL SECTION}

Materials. Synthesis and growth of triarylamine TAA 1 nanowires by electropolymerization were carried out following the procedures described previously. ${ }^{26,29} \mathrm{~A} 1.25 \times 10^{7} \mathrm{~V} / \mathrm{m}$ applied DC electric 
field was used, as it was shown to be optimal for the alignment of nanowires during the supramolecular polymerization of TAA $1 .{ }^{26}$ The samples were prepared on special glass substrates, composed of 15 interdigitated gold micro-electrodes pairs of $100 \mathrm{~nm}$ height, $2.5 \mathrm{~mm}$ long, having each a width of $10 \mu \mathrm{m}$ and spaced away by $10 \mu \mathrm{m}$. The GIWAXS data were collected post-process. In all the manuscript "in-situ" refers to the process of fabrication, meaning that nanowires were synthesized in the final device. The substrates with their microelectrodes were mounted on a precise goniometer platform allowing the alignment and movement of the sample according to six degrees of freedom with a very good repeatability (full vertical axis rotation: $1 \mathrm{mdeg}$ ). ${ }^{27}$ In the configuration where the incident beam is parallel to the microelectrodes $(\omega=0$, Fig. 1$)$, the misalignment (non-parallelism) of the incident $\mathrm{x}$-ray beam with the $2.5 \mathrm{~mm}$ - long electrodes is estimated to be less than 0.3 degrees.

GIWAXS experiments. The experiments were conducted at room temperature on the SIRIUS beamline at the French National Synchrotron Radiation facility SOLEIL in Saint-Aubin, France. ${ }^{27}$ An X-ray energy of $10 \mathrm{keV}$ (X-ray wavelength $1.24 \AA$ ) was selected with a Si(111) double crystals monochromator. The beam size $500 \times 100 \mu \mathrm{m}^{2}(\mathrm{H} \times \mathrm{V})$ was obtained using a two-slit system. A beam stop was used to stop direct and reflected beams. Two-dimensional x-ray scattering data were collected on a DECTRIS PILATUS3 1M 2D detector positioned $472 \mathrm{~mm}$ downstream of the sample. The diffraction pattern of silver behenate was used to calibrate the sample-to-detector distance. ${ }^{40}$ Two laterally rotated detector positions were used for covering the scattering vector range up to $2 \AA^{-1}$. Patterns were corrected from silica glass and air scattering by subtracting normalized background obtained from empty cell. For the data presented, the incidence angle was set at $0.12^{\circ}$, below the total external reflection critical angle of the glass substrate. Typical exposure times for these samples ranged from 1 to $20 \mathrm{~min}$. Because $q_{\mathrm{z}}$ is not probed for more than the very 
shallow exit angles, the illuminated detector pixels do not linearly correspond to the examined $q$ space. Therefore a new mesh was generated and processed starting from the raw image of the 2D detector to lead to the $q$-map shown in the GIWAXS patterns (Fig. 2, Fig. S1). The intensity $I(q)$ profiles were obtained from background subtracted images using home-developed software, through re-sampling to polar $I(q, \phi)$ coordinates and renormalization to entire integration range going from $\phi=0^{\circ}$ to $\phi=90^{\circ}$.

Measurement of Bragg reflection intensities. Accurate subtraction of background is important to obtain accurate intensity measurements. A first approach used a global background function expanded as a polynomial. However, the scattered background is not circularly symmetric. Thus, to obtain reliable radial and azimuthal profiles of the Bragg reflections, two-dimensional background level matrices were obtained from peripheral regions of reflection arcs using series of one-dimensional ring profiles. This was particularly advantageous for estimating the radial profiles when there is little space between close arc-shaped reflections of adjacent Bragg spots.

For an ensemble of nanowires exhibiting preferred orientation, the scattered intensities are modeled as an incoherent sum of intensities from the individual objects. The intensity at a given point in reciprocal space having spherical coordinates $\left(q_{i}, \psi_{i}, \sigma_{i}\right)$ results from the product of weighting functions accounting for radial peak broadening (in $q$, distribution function $G_{q}$ centered at the maximum $q_{i}$ with width $\Delta q_{i}$ ) and preferred orientation (functions $G \Psi$ and $G_{\sigma}$ centered at the maxima $\psi_{i}$ and $\sigma_{i}$ and having widths $\Delta \psi_{i}$ and $\Delta \sigma_{i}$ respectively). Summing the weighted contributions to $I_{i}$ from all reciprocal lattice points $n$ (provided that they intersect the Ewald sphere), the expression for the intensity reads

$$
I_{i}\left(q_{i}, \psi_{i}, \sigma_{i}\right) \propto \sum_{n} C_{\mathrm{corr}} G_{q}\left(q_{i}-q_{n}, \Delta_{q_{i}}\right) G_{\psi}\left(\psi_{i}-\psi_{n}, \Delta_{\psi_{i}}\right) G_{\sigma}\left(\sigma_{i}-\sigma_{n}, \Delta_{\sigma_{i}}\right)
$$


where $C_{\text {corr }}$ contains various geometric corrections in the GIWAXS pattern obtained with a sample tilted by an angle $\theta_{0}$ (in the present experiments, $\theta_{0}=0.12^{\circ}$ ) with respect to the incoming beam (see Fig.1e):

$$
C_{\text {corr }}=\left[p_{\mathrm{H}}\left(1-\sin ^{2} \mu \cos ^{2} \delta\right)+\left(1-p_{\mathrm{H}} \sin ^{2} \delta\right)\right] \frac{1-e^{-\mu_{a} T\left(\frac{1}{\sin \theta_{0}}+\frac{1}{\sin \delta}\right)}}{\mu_{a} T\left(\frac{1}{\sin \theta_{0}}+\frac{1}{\sin \delta}\right)}
$$

The first factor is the polarization factor ${ }^{41,42}$ at the linearly polarized SIRIUS beamline at the SOLEIL synchrotron (the fraction of radiation that is polarized in the horizontal direction, $p_{\mathrm{H}}$, is larger than 98\%), and the second factor is the absorbance correction. Here, $2 \theta$ designates the total scattering angle, while the angles $\mu$ and $\delta$ define the direction of the diffracted beam with respect to the incident beam: $\mu$ is the horizontal ("longitude") angle and $\delta$ the vertical ("latitude") angle of the intersection of the diffracted beam with the Ewald sphere of reflection. In this experimental work, the detector is vertical, and thereby the calculation of $\mu$ depends on the angular position of the detector, while $\delta$ does not. The absorbance correction results from the longer path of x-rays at low exit angles than at high angles, through a film of thickness $T$, assumed here to have a uniform absorbance. Nevertheless, as the sample film is very thin $(T \sim 100 \mathrm{~nm})$, with an extinction coefficient $\mu_{a} \sim 2 \mathrm{~mm}^{-1}$ for a wavelength of $1.24 \AA$ (calculated from the chemical composition and tabulated atomic values), this absorption correction is negligibly small.

\section{ASSOCIATED CONTENT}

Supporting information. Additional GIWAXS patterns at high concentration are presented.

\section{AUTHOR INFORMATION}




\section{Corresponding Authors:}

Nicolas Giuseppone - Institut Charles Sadron, Université de Strasbourg, C.N.R.S., UPR22, 23 rue du Loess, 67034 Strasbourg, France; Email: giuseppone@unistra.fr ; ORCID: 0000-00034093-3000

Patrick Kékicheff - Institut Charles Sadron, Université de Strasbourg, C.N.R.S., UPR22, 23 rue du Loess, 67034 Strasbourg, France; Synchrotron SOLEIL, Saint Aubin, L'Orme des Merisiers, BP48, 91192 Gif-sur-Yvette, France; Email: patrick.kekicheff@ics-cnrs.unistra.fr ; ORCID: 0000$0001-8465-9627$

\section{Authors Adress:}

Melodie Galerne - Institut Charles Sadron, Université de Strasbourg, C.N.R.S., UPR22, 23 rue du Loess, 67034 Strasbourg, France ; ORCID: 0000-0002-8535-2923

Benoît Heinrich - Institut de Physique et Chimie des Matériaux de Strasbourg, Université de Strasbourg, C.N.R.S., UMR7504, 23 rue du Loess, 67034 Strasbourg, France; ORCID: 00000001-6795-2733

Arnaud Hemmerle - Synchrotron SOLEIL, Saint Aubin, L'Orme des Merisiers, BP48, 91192 Gifsur-Yvette, France ; ORCID : 0000-003-1722-2024

Philippe Fontaine - Synchrotron SOLEIL, Saint Aubin, L'Orme des Merisiers, BP48, 91192 Gifsur-Yvette, France ; ORCID : 0000-0003-3394-6508 


\section{Author Contributions}

N.G. conceived the supramolecular electropolymerization; M.G. prepared the samples; P.K. and P.F. conceived and with B.H. planned the GIWAXS experiments; A.H. and P.F. aligned the SIRIUS beamline at SOLEIL synchrotron; B.H. and P.K. performed, analyzed and interpreted the results; P.K., M.G. and N.G. wrote the paper; all authors commented, reviewed the manuscript and gave approval to the final version of the manuscript.

\section{Notes}

The authors declare no competing financial interest.

\section{ACKNOWLEDGMENT}

We thank SOLEIL synchrotron for beamtime provision. Experiments were carried out within the framework of the MICASOL platform set between Institut Charles Sadron, C.N.R.S., Strasbourg, France, and SOLEIL synchrotron (www.ics-cnrs.unistra.fr/micasol). Part of the experimental work (sample preparation) was performed within the framework of the IRTG “Soft Matter Science: Concepts for the Design of Functional Materials” (M.G. and N.G.).

\section{REFERENCES}

(1) Schenning, A. P. H. J.; Meijer, E. W. Supramolecular electronics; nanowires from selfassembled $\pi$-conjugated systems. Chem. Comm. 2005, 26, 3245-58. 
(2) Su, B.; Wu, Y.; Jiang, L. The Art of Aligning One-Dimensional (1D) Nanostructures. Chem. Soc. Rev. 2012, 41, 7832-7856.

(3) Jain, A.; George, S. J. New directions in supramolecular electronics. Materials Today 2015, 18, 206-214.

(4) Moulin, E.; Cid, J. J.; Giuseppone, N. Advances in Supramolecular Electronics - From Randomly Self-Assembled Nanostructures to Addressable Self-Organized Interconnects. $A d v$. Mater. 2013, 25, 477-487.

(5) Jo, G.; Jeong, J. W.; Choi, S.; Kim, H.; Park, J. J.; Jung, J.; Chang, M. Large-Scale Alignment of Polymer Semiconductor Nanowires for Efficient Charge Transport via Controlled Evaporation of Confined Fluids. ACS Appl. Mater. Interfaces 2019, 11, 1135-1142.

(6) Chaudhary, V.; Pandey, R. K.; Prakash, R.; Kumar, N.; Singh, A. K. Highly Aligned and Crystalline Poly(3-Hexylthiophene) Thin Films by off-Center Spin Coating for High Performance Organic Field-Effect Transistors. Synth. Met. 2019, 258, 116221.

(7) Chang, M. Choi, D.; Egap, E. Macroscopic Alignment of One-Dimensional Conjugated Polymer Nanocrystallites for High-Mobility Organic Field-Effect Transistors. ACS Appl. Mater. Interfaces 2016, 8, 13484-13491.

(8) Xiang, J.; Lu, W.; Hu, Y.; Wu, Y.; Yan, H.; Lieber, C. M. Ge/Si Nanowire Heterostructures as High-Performance Field-Effect Transistors. Nature 2006, 441, 489-493.

(9) Awartani, O.; Kudenov, M. W.; Kline, R. J.; O’Connor, B. T. In-Plane Alignment in Organic Solar Cells to Probe the Morphological Dependence of Charge Recombination. Adv. Funct. Mater. 2015, 25, 1296-1303.

(10) Yang, Y.; Mielczarek, K.; Zakhidov, A.; Hu, W. Effects of Nanostructure Geometry on Polymer Chain Alignment and Device Performance in Nanoimprinted Polymer Solar Cell. Org. Photonic Mater. Devices XV 2013, 8622, 862211.

(11) Yang, Z.; Chen, T.; He, R.; Guan, G.; Li, H.; Qiu, L.; Peng, H. Aligned Carbon Nanotube Sheets for the Electrodes of Organic Solar Cells. Adv. Mater. 2011, 23, 5436-5439. (12) Peng, Q.; Wen, Z.; Zeng, G.; Zou, J. Lu, S. Aligned Coaxial Nanowires of Carbon Nanotubes Partially Sheathed with Polyaniline for Chemical Sensors. Chem. Lett. 2009, 38, $380-381$. 
(13) Mcalpine, M. C.; Ahmad, H.; Wang, D.; Heath, J. R. Highly Ordered Nanowire Arrays on Plastic Substrates for Ultrasensitive Flexible Chemical Sensors. Nat. Mater., 2007, 6, 379384.

(14) Persson, N. E.; Engmann, S.; Richter, L. J.; Delongchamp, D. M. In Situ Observation of Alignment Templating by Seed Crystals in Highly Anisotropic Polymer Transistors. Chem. Mater. 2019, 31, 4133-4147.

(15) Nahid, M. M.; Gann, E.; Thomsen, L.; McNeill, C. R. NEXAFS Spectroscopy of Conjugated Polymers. Eur. Polym. J. 2016, 81, 532-554.

(16) Zarubin, V. A.; Li, T.-De; Humagain, S.; Ji, H.; Yager, K. G.; Greenbaum, S. G.; Vuong, L. T. Improved Anisotropic Thermoelectric Behavior of Poly(3,4Ethylenedioxythiophene):Poly(Styrenesulfonate) via Magnetophoresis. ACS Omega 2018, 3, $12554-12561$.

(17) Lin, Y.-W.; Lin, C.-J.; Chou, Y.-H.; Liu, C.-L.; Chang, H.-C.; Chen, W.-C. Nonvolatile Organic Field Effect Transistor Memory Devices Using One-Dimensional Aligned Electrospun Nanofiber Channels of Semiconducting Polymers. J. Mater. Chem. C 2013, 1, $5336-5343$.

(18) Bastianini, F.; Pérez, G. E.; Hobson, A. R.; Rogers, S. E.; Parnell, A. J.; Grell, M.; Gutiérrez, A. F.; Dunbar, A. D. F. In-situ Monitoring Poly(3-Hexylthiophene) Nanowire Formation and Shape Evolution in Solution via Small Angle Neutron Scattering. Sol. Energy Mater. Sol. Cells 2019, 202, 110128.

(19) Weller, T.; Rundel, K.; Krauss, G.; McNeill, C. R.; Thelakkat, M. Highly Efficient and Balanced Charge Transport in Thieno[3,4- c] Pyrrole-4,6-Dione Copolymers: Dramatic Influence of Thieno[3,2- b] Thiophene Comonomer on Alignment and Charge Transport. $J$. Phys. Chem. C 2018, 122, 7565-7574.

(20) Gujral, A.; O’Hara, K. A.; Toney, M. F.; Chabinyc, M. L.; Ediger, M. D. Structural Characterization of Vapor-Deposited Glasses of an Organic Hole Transport Material with XRay Scattering. Chem. Mater. 2015, 27, 3341-3348.

(21) Moulin, E.; Armao, J. J. IV; Giuseppone, N. Triarylamyne-Based Supramolecular Polymers: Structure, Dynamics and Functions. Acc. Chem. Res. 2019, 52, 975-983. 
(22) Faramarzi, V.; Niess, F.; Moulin, E.; Maaloum, M.; Dayen, J. F.; Beaufrand, J. B.; Zanettini, S.; Doudin, B.; Giuseppone, N. Light-Triggered Self-Construction of Supramolecular Organic Nanowires as Metallic Interconnects. Nat. Chem. 2012, 4, 485-490. (23) Armao, J. J.; Rabu, P.; Moulin, E.; Giuseppone, N. Long-Range Energy Transport via Plasmonic Propagation in a Supramolecular Organic Waveguide. Nano Lett. 2016, 16, 28002805.

(24) Armao, J. J.; Domoto, Y.; Umehara, T.; Maaloum, M.; Contal, C.; Fuks, G.; Moulin, E.; Decher, G.; Javahiraly, N.; Giuseppone, N. Supramolecular Organic Nanowires as Plasmonic Interconnects. ACS Nano 2016, 10, 2082-2090.

(25) Busseron, E.; Cid, J.-J.; Wolf, A.; Du, G.; Moulin, E.; Fuks, G.; Maaloum, M.; Polavarapu, P.; Ruff, A.; Saur, A.-K.; Ludwigs, S.; Giuseppone, N. Light-Controlled Morphologies of Self-Assembled Triarylamine-Fullerene Conjugates. ACS Nano, 2015, 9, $2760-2772$.

(26) Ellis, T. K.; Galerne, M.; Armao, J. J. IV; Osypenko, A.; Martel, D.; Maaloum, M.; Fuks, G.; Gavat, O.; Moulin, E.; Giuseppone, N. Supramolecular Electropolymerization. Angew. Chem. Int. Ed. 2018, 57, 15749-15753.

(27) Fontaine, P.; Ciatto, G.; Aubert, N.; Goldmann, M. Soft interfaces and resonant investigation on undulator source: A surface X-ray scattering beamline to study organic molecular films at the SOLEIL synchrotron. Sci. Adv. Mater. 2014, 6, 2312-2316.

(28) International tables for Crystallography, edited by T. Hahn, Dordrecht, London: Published for the International Union of Crystallography by Kluwer Academic Publishers, 2002.

(29) Nyrkova, I.; Moulin, E.; Armao IV, J. J.; Maaloum, M.; Heinrich, B.; Rawiso, M.; Niess, F.; Cid, J.-J.; Jouault, N.; Buhler, E.; Semenov, A. N.; Giuseppone, N. Supramolecular Self-Assembly and Radical Kinetics in Conducting Self-Replicating Nanowires. ACS Nano 2014, $8,10111-10124$.

(30) Kékicheff, P.; Cabane, B. Crystallography of Systems with Long Periods: a NeutronScattering Study of Sodium Dodecyl Sulfate/Water Mesophases. Acta Cryst. 1988, B44, 395406.

(31) Kékicheff, P. From cylinders to bilayers: A structural study of phase transformations in a lyotropic liquid crystal. Mol. Cryst. Liq. Cryst. 1991, 198, 131-144. 
(32) McGrath, K. M.; Kékicheff, P.; Kléman, M. Spiral textures in lyotropic liquid crystals: first order transition between normal hexagonal and lamellar gel phases. J. Phys. II France 1993, 3, 903-926.

(33) Stein, R. S.; Wilkes, G. L. Structure and Properties of Oriented Polymers (Materials Science Series), ed. Ward, I. M., John Wiley \& Sons, London: Applied Science, 1975, p. 57. (34) Lowell, R.; Mitchell, G. R. Molecular orientation distribution derived from an arbitrary reflection. Acta Cryst. 1981, A37, 135-137.

(35) Millane, R. P. Relating reflection boundaries in X-ray fiber diffraction patterns to specimen morphology and their use for intensity measurement. J. Macromol. Sci. Phys., 1989, B28, 149-166.

(36) Fraser, R. D. B.; Macrae, T. P.; Miller, A.; Rowlands, R. J. Digital processing of fibre diffraction patterns. J. Appl. Cryst. 1976, 9, 81-94.

(37) Yoneda, Y. Anomalous surface reflection of X-rays. Phys. Rev. 1963, 131, 2010-2013.

(38) Langford, J. I.; Wilson, A. J. C. Scherrer after sixty years: A survey and some new results in the determination of crystallite size. J. Appl. Cryst. 1978, 11, 102-113.

(39) Smilgies, D.-M., Scherrer grain-size analysis adapted to grazing-incidence scattering with area detectors. J. Appl. Cryst. 2009, 42, 1030-1034.

(40) Nyam-Osor, M.; Soloviov, D. V.; Kovalev, Yu. S.; Zhigunov, A.; Rogachev, A. V.; Ivankov, O. I.; Erhan, R. V.; Kuklin, A. I. Silver behenate and silver stearate powders for calibration of SAS instruments. J. Phys.: Conf. Ser. 2012, 351, 012024.

(41) Smilgies, D.-M. Geometry-independent intensity correction factors for grazingincidence diffraction. Rev. Sci. Instrum. 2002, 73, 1706-1710.

(42) Schlepütz, C. M.; Herger, R.; Willmott, P. R.; Patterson, B. D.; Bunk, O.; Brönnimann, C.; Henrich, B.; Hülsen, G.; Eikenberry, E. F. Improved data acquisition in grazing-incidence X-ray scattering experiments using a pixel detector. Acta Crystallogr. 2005, A61, 418-425. 